Computer Vision, Graphics, and Pattern Recognition Group Department of Mathematics and Computer Science University of Mannheim 68131 Mannheim, Germany

Reihe Informatik

$8 / 2001$

Analysis of Optical Flow Models in the
Framework of Calculus of Variations

Walter Hinterberger, Otmar Scherzer, Christoph Schnörr, and Joachim Weickert

Technical Report 8/2001

Computer Science Series

April 2001

The technical reports of the CVGPR Group are listed under http://www.cvgpr .uni-mannheim. de/publications .html 


\title{
Analysis of Optical Flow Models in the Framework of Calculus of Variations
}

\author{
Walter Hinterberger ${ }^{1}$, Otmar Scherzer ${ }^{2}$, \\ Christoph Schnörr ${ }^{3}$, and Joachim Weickert ${ }^{3}$ \\ ${ }^{1}$ Mathconsult $\mathrm{GmbH}$ \\ Altenberger Str. 64 \\ A-4040 Linz, Austria \\ walter@mathconsult .co.at \\ ${ }^{2}$ Angewandte Mathematik \\ Universität Bayreuth \\ D-95440 Bayreuth, Germany \\ otmar.scherzer@uni-bayreuth.de \\ ${ }^{3}$ Computer Vision, Graphics, and Pattern Recognition Group \\ Department of Mathematics and Computer Science \\ University of Mannheim \\ D-68131 Mannheim, Germany \\ \{Christoph.Schnoerr, Joachim.Weickert\}@uni-mannheim.de
}

\begin{abstract}
In image sequence analysis, variational optical flow computations require the solution of a parameter dependent optimization problem with a data term and a regularizer. In this paper we study existence and uniqueness of the optimizers. Our studies rely on quasiconvex functionals on the spaces $W^{1, p}\left(\Omega, \mathbb{R}^{d}\right)$, with $p>1$, $B V\left(\Omega, \mathbb{R}^{d}\right), B D(\Omega)$. The methods that are covered by our results include several existing techniques. Experiments are presented that illustrate the behavior of these approaches.
\end{abstract}

Keywords: Optical flow, calculus of variations, quasiconvex functionals, functions of bounded variation and deformation

\section{Introduction}

Optical flow is the apparent 2D motion that needs to be recovered from a video sequence. $2 \mathrm{D}$ motions find diverse applications in video processing as well as in computer vision. In video compression knowledge of motion helps to remove temporal data redundancy which in turn is used to compress video sequences with high compression ratios.

For the detection of motion one typically uses the following model. Let $I(x, t)$ be the measured image intensity at position $(x, t)$, with $x=\left(x_{1}, x_{2}\right)$ in $[0,1]^{2} \subseteq \mathbb{R}^{2}$. Let $x(t)$ 
be the parametrisation of a path of constant image intensity, i.e., $I(x(t), t)=$ constant for $t \in[0, \infty)$, then

$$
\frac{\partial I}{\partial t}(x(t), t)=0
$$

By applying the chain rule and assuming that structures do not change their intensities over time, (1) can be written as the optical flow equation

$$
\begin{aligned}
0 & =I_{x_{1}}(x, t) u(x, t)+I_{x_{2}}(x, t) v(x, t)+I_{t}(x, t) \\
& =(\nabla I)^{t}(x, t) \vec{w}(x, t)+I_{t}(x, t),
\end{aligned}
$$

where $\vec{w}(x, t)=(u, v)^{t}(x, t)$ denotes the optical flow field. We use the convention that subscripts denote partial derivatives and $\frac{\partial}{\partial t}$ denotes the total derivative.

In this paper we analyze models for recovering a motion field $\vec{w}$ in (2) from a sequence of image intensities.

\section{Models for motion representation}

The motion field $\vec{w}=(u, v)^{t}$ is not uniquely determined by (2), since it is one equation for two unknown functions $u$ and $v$. Thus additional constraints have to imposed and there have been proposed several models in the literature.

Variational optical flow computations started with the pioneering work of Horn and Schunck [19] who proposed to calculate an approximate solution of (2) that minimizes the functional

$$
J_{\mathrm{HS}}(\vec{w})=\frac{1}{2} \int_{\Omega}\left(|\nabla u(x)|^{2}+|\nabla v(x)|^{2}\right) d x
$$

Recently there has been a trend to use more sophisticated constraints to preserve edges and corners in the motion field (see e.g. [23, 8, 27, 30, 2, 31]). This can be achieved by considering e.g. penalizing functionals like

$$
J_{\mathrm{NE}}(\vec{w})=\int_{\Omega} \operatorname{trace}\left((\nabla \vec{w}(x))^{t} D_{2}(\nabla I)(x)(\nabla \vec{w}(x))\right) d x
$$

with

$$
D_{2}(\nabla I)(x)=\frac{1}{|\nabla I(x)|^{2}+2 \lambda^{2}}\left\{\left(\begin{array}{c}
\frac{\partial I}{\partial x_{2}}(x) \\
-\frac{\partial I}{\partial x_{1}}(x)
\end{array}\right)\left(\begin{array}{c}
\frac{\partial I}{\partial x_{2}}(x) \\
-\frac{\partial I}{\partial x_{1}}(x)
\end{array}\right)^{t}+\lambda^{2} E\right\} .
$$

Here and in the sequel of the paper $|$.$| denotes the Euclidean norm and E$ denotes the unitary matrix. The motivation for using such penalizing functionals comes from anisotropic diffusion filtering. For some background on this topic we refer to [29].

Another frequently used edge preserving technique is via $B V$ penalizing functionals like [8]

$$
J_{\mathrm{BV}}(\vec{w})=\int_{\Omega}(|\nabla u(x)|+|\nabla v(x)|) d x
$$


where both $\int_{\Omega}|\nabla u| d x$ and $\int_{\Omega}|\nabla v| d x$ are understood as the bounded variations seminorms of $u$ and $v$. For a definition of the space of functions of bounded variation and the semi-norm we refer to [13].

Following the standard way of solving a constraint optimization problems, we approximate minimizers of $J_{\mathrm{HS}}, J_{\mathrm{NE}}, J_{\mathrm{BV}}$ that satisfy the constraint (2) by solutions of the unconstraint optimization problem to minimize the functional

$$
J_{i}^{\lambda}(\vec{w})=\lambda \int_{\Omega} \phi\left(\left((\nabla I)^{t} \vec{w}+I_{t}\right)^{2}\right) d x+J_{i}(\vec{w})
$$

where $\lambda$ is the positive penalizing parameter and $i=H S, N E, B V$, respectively. Here $\phi$ is a nonnegative function. Examples of frequently used functions $\phi$ in optical flow computations are $\phi()=.|\cdot|^{p}, p=1,2$.

\section{Lower semi-continuous functionals}

For the analysis of optical flow problems we utilize classical result of calculus of variations and nonlinear partial differential equation. All the particular functionals for optical flow computations outlined in Section 2 fit into the class of problems of minimization of a functional

$$
\vec{w} \rightarrow I[\vec{w}]:=\int_{\Omega} f(x, \vec{w}, \nabla \vec{w}) d x .
$$

In this particular paper we consider minimization over Banach spaces $W^{1, p}\left(\Omega, \mathbb{R}^{d}\right)$ and $B V\left(\Omega, \mathbb{R}^{d}\right), d \geq 1$. All along the remainder of this paper we assume that $\Omega \subseteq \mathbb{R}^{n}$ is bounded with piecewise smooth boundary.

In the first case we can utilize classical results on calculus of variations (see e.g., Morrey [21, 22], Ball [5], Dacorogna [10]) which guarantee lower semi-continuity of the functional $I[\vec{w}]$ in $W^{1, p}\left(\Omega, \mathbb{R}^{d}\right), 1<p \leq \infty$. These abstract results will be applied afterwards to prove existence of minimizers of optical flow models. Weak lower semicontinuity of $I[\vec{w}]$ on $B V\left(\Omega, \mathbb{R}^{d}\right)$ is a rather challenging topic. There are several results in this direction (see e.g. $[4,15,16,14]$ to name but a few) which deal with semicontinuity of $I[\vec{w}]$ in a general setting. There are some easier results available if we take into account the special structure of penalized least squares functionals such as modeled in Section 2.

\subsection{Quasiconvex functional on $W^{1, p}\left(\Omega, \mathbb{R}^{d}\right)$}

In this section we recall the concept of quasiconvexity and summarize some results on (weak) lower semi-continuity of the functional (7) on the space $W^{1, p}\left(\Omega, \mathbb{R}^{d}\right)$. We associate with each space of vector-valued functions $X\left(\Omega, \mathbb{R}^{d}\right)$ the norm

$$
\|\vec{f}\|:=\sqrt{\sum_{i=1}^{d}\left\|f_{i}\right\|_{X}^{2}} .
$$


Definition 1. A function

$$
\begin{aligned}
f: \mathbb{R}^{n \times d} & \rightarrow \mathbb{R} \\
P & \rightarrow f(P)
\end{aligned}
$$

is called quasiconvex if

$$
\int_{\Omega} f(P) d y \leq \int_{\Omega} f(P+\nabla \vec{v}) d y
$$

for each $P \in \mathbb{R}^{n \times d}$ and $\vec{v} \in C_{c}^{\infty}\left(\Omega ; \mathbb{R}^{d}\right)$. Here $C_{c}^{\infty}\left(\Omega ; \mathbb{R}^{d}\right)$ denotes the subspace of $C^{\infty}\left(\Omega ; \mathbb{R}^{d}\right)$ with functions of compact support in $\Omega$.

Note that any convex function is quasiconvex (see e.g [12]).

Sometimes instead of (8) an equivalent formulation of quasiconvexity is used

$$
f(P) \leq \frac{1}{\operatorname{meas}(\Omega)} \int_{\Omega} f(P+\nabla \vec{v}) d y .
$$

The following structural theorem holds for quasiconvex functionals (see e.g. [12]).

Theorem 2. Let $1<p<\infty$. Suppose $f$ satisfies

$$
0 \leq f(P) \leq C\left(1+|P|^{p}\right) \quad\left(P \in \mathbb{R}^{n \times d}\right),
$$

for some constants $C$ and $p>1$; here $|P|$ denotes the Frobenius norm of the matrix $P$. Then the functional

$$
G[\vec{w}]=\int_{\Omega} f(\nabla \vec{w}) d x
$$

is lower semi-continuous with respect to weak convergence in $W^{1, p}\left(\Omega, \mathbb{R}^{d}\right)$ if and only if $f$ is quasiconvex.

Now we turn to the more general situation that $f$ is of the general form (7). We can rely on a variety of results. A few of them are quoted here for the readers convenience.

One of the first results in this direction can be found in Morrey [21]. More recently Fonseca and Müller [15] proved the following result:

Theorem 3. (Fonseca and Müller) Let $f$ be continuous from $\Omega \times \mathbb{R}^{d} \times \mathbb{R}^{n \times d}$ into $[0, \infty)$ satisfying

1. $f(x, \vec{w}, \cdot)$ is quasiconvex

2. There exits a nonnegative, bounded, continuous function $\bar{f}: \Omega \times \mathbb{R}^{d} \rightarrow[0, \infty)$, constants $\underline{\alpha}, \bar{\alpha}>0$ such that

$$
\underline{\alpha} \bar{f}(x, \vec{w})|P|-\bar{\alpha} \leq f(x, \vec{w}, P) \leq \bar{\alpha} \bar{f}(x, \vec{w})(1+|P|)
$$

for all $(x, \vec{w}, P) \in \Omega \times \mathbb{R}^{d} \times \mathbb{R}^{n \times d}$. 
3. For all $\left(x_{0}, \vec{w}_{0}\right) \in \Omega \times \mathbb{R}^{d}$ and for all $\eta>0$ there exists $\delta>0$ such that for $\left|x-x_{0}\right|+\left|\vec{w}-\vec{w}_{0}\right| \leq \delta$ implies that

$$
f\left(x_{0}, \vec{w}, P\right)-f\left(x_{0}, \vec{w}_{0}, P\right) \geq-\eta(1+|P|),
$$

and

$$
\left|f\left(x_{0}, \vec{w}, P\right)-f(x, \vec{w}, P)\right| \leq \eta(1+|P|)
$$

Then if $\vec{w}_{n}, \vec{w} \in W^{1,1}\left(\Omega, \mathbb{R}^{d}\right)$ and $\vec{w}_{n} \rightarrow \vec{w}$ in $L^{1}\left(\Omega, \mathbb{R}^{d}\right)$, then

$$
I[\vec{w}] \leq \liminf _{n \rightarrow \infty} I\left[\vec{w}_{n}\right] .
$$

For our purposes Theorem 2 is not practicable since for optical flow simulations we require instead of (12) estimates of the form

$$
\underline{\alpha}(1+|\vec{w}|+|P|) \leq f(x, \vec{w}, P) \leq \bar{\alpha}(1+|\vec{w}|+|P|)
$$

on the space of functions of bounded variation (instead of $W^{1,1}\left(\Omega, \mathbb{R}^{d}\right)$ ). Modifications of this theorem which can be applied to the analysis of optical flow problems are given below.

In Dacorogna [10, p. 167] we find the following result:

Theorem 4. (see Dacorogna) For $1<p<\infty$. Let $f$ be continuous from $\Omega \times \mathbb{R}^{d} \times$ $\mathbb{R}^{n \times d}$ into $[0, \infty)$ satisfying

1. $f(x, \vec{w}, \cdot)$ is quasiconvex.

2. There exists a positive constant $\bar{\alpha}$ such that

$$
0 \leq f(x, \vec{w}, P) \leq \bar{\alpha}\left(1+|\vec{w}|^{p}+|P|^{p}\right)
$$

3. There exists a positive constant $\beta>0$ such that

$$
\begin{aligned}
\left|f\left(x, \vec{w}, P_{1}\right)-f\left(x, \vec{w}_{0}, P_{2}\right)\right| \leq \beta & \left(1+|\vec{w}|^{p-1}+\left|\vec{w}_{0}\right|^{p-1}+\left|P_{1}\right|^{p-1}+\left|P_{2}\right|^{p-1}\right) \\
& \cdot\left(\left|\vec{w}-\vec{w}_{0}\right|+\left|P_{1}-P_{2}\right|\right) .
\end{aligned}
$$

4. There exists a continuous, increasing function $\eta$ satisfying $\eta(0)=0$ such that

$$
|f(x, \vec{w}, P)-f(y, \vec{w}, P)| \leq \eta(|x-y|)\left(1+|\vec{w}|^{p}+|P|^{p}\right) .
$$

Then $I[\vec{w}]$ is weakly lower semi-continuous on $W^{1, p}\left(\Omega ; \mathbb{R}^{d}\right)$.

The following corollary can be proven similarly to Theorem 3 by taking into account the Sobolev embedding theorem. 
Corollary 5. For $1<p<\infty$ and $q$ satisfying

$$
\begin{array}{cll}
1 \leq q<\frac{n p}{n-p} & \text { if } & 1<p<n \\
& \text { or } & \\
1 \leq q<\infty & \text { if } & p \geq n
\end{array}
$$

set $\bar{s}:=\max \{q, p\}$. Moreover, let $f$ be continuous from $\Omega \times \mathbb{R}^{d} \times \mathbb{R}^{n \times d}$ into $[0, \infty)$ satisfying

1. $f(x, \vec{w}, \cdot)$ is quasiconvex.

2. There exists a positive constant $\bar{\alpha}$ such that

$$
0 \leq f(x, \vec{w}, P) \leq \bar{\alpha}\left(1+|\vec{w}|^{\bar{s}}+|P|^{p}\right) .
$$

3. There exists a positive constant $\beta>0$ such that

$$
\begin{gathered}
\left|f\left(x, \vec{w}, P_{1}\right)-f\left(x, \vec{w}_{0}, P_{2}\right)\right| \leq \beta\left(1+|\vec{w}|^{\bar{s}-1}+\left|\vec{w}_{0}\right|^{\bar{s}-1}+\left|P_{1}\right|^{p-1}+\left|P_{2}\right|^{p-1}\right) \\
\cdot\left(\left|\vec{w}-\vec{w}_{0}\right|+\left|P_{1}-P_{2}\right|\right) .
\end{gathered}
$$

4. There exists a continuous, increasing function $\eta$ satisfying $\eta(0)=0$ such that

$$
|f(x, \vec{w}, P)-f(y, \vec{w}, P)| \leq \eta(|x-y|)\left(1+|\vec{w}|^{\bar{s}}+|P|^{p}\right) .
$$

Then $I[\vec{w}]$ is weakly lower semi-continuous on $W^{1, p}\left(\Omega ; \mathbb{R}^{d}\right)$.

\subsection{Quasiconvex functional on $B V\left(\Omega, \mathbb{R}^{d}\right)$}

If $f(x, \vec{w}, \cdot)$ is quasiconvex then the functional (7) defined on $B V\left(\Omega, \mathbb{R}^{d}\right)$ is implicitly defined via the following limiting procedure (relaxation)

$$
\begin{aligned}
I[\vec{w}]:=\inf _{\left\{\vec{w}_{n}\right\}}\left\{\liminf _{n \rightarrow \infty} \int_{\Omega} f\left(x, \vec{w}_{n}(x), \nabla \vec{w}_{n}(x)\right) d x:\right. \\
\left.\vec{w}_{n} \in W^{1,1}\left(\Omega, \mathbb{R}^{d}\right) \text { and } \vec{w}_{n} \rightarrow \vec{w} \in L^{1}\left(\Omega, \mathbb{R}^{d}\right)\right\} .
\end{aligned}
$$

If $f$ is quasiconvex and satisfies some growth properties then there exists an integral representation for $I[\vec{w}]$ consisting of three integrals (see e.g. $[15,16]$ ); the first integral takes care of the regular parts of $\nabla \vec{w}$ and the second and third parts take care of the singular parts of the measure $\nabla \vec{w}$.

A few results are available in the literature on weak lower semi-continuity of $I[\vec{w}]$ on $B V\left(\Omega, \mathbb{R}^{d}\right)$. One result showing semi-continuity of this functional has been given in Aviles and Giga [4]. Their result is technically complicated and we confine our 
considerations to a subclass of functionals (7) on the space of functions of bounded variation for which an easier analysis is accessible. Let us assume bounded variation penalization models of the form

$$
f(x, \vec{w}, \nabla \vec{w})=\phi(x, \vec{w})+|\nabla \vec{w}|_{1},
$$

where for $\vec{w}=\left(w^{1}, \cdots, w^{d}\right)^{t}$

$$
|\nabla \vec{w}|_{1}=\sum_{i=1}^{d}\left|\nabla w^{i}\right|
$$

That is we assume that the functional $f$ consists of two sums, where only one is dependent on the gradient. In this situation lower semi-continuity of the functional $I[w]$ is easier tractable. At the current status of research in optical flow models on the space of functions of bounded variation it is sufficient to consider such models; all (numerically) investigated models are of such a form. The technical advantage of the term $|\nabla \vec{w}|_{1}$ is that the relaxed functional $\int_{\Omega}|\nabla \vec{w}|_{1} d x$ is the bounded variation semi-norm of all components for which an integral decomposition in regular and singular parts of the measure is well-understood (see e.g. [13]).

In the following we utilize a weak continuity result on $L^{p}\left(\Omega, \mathbb{R}^{d}\right)$ similar to one stated in Dacorogna [9]. Surprisingly we were not able to find the particular result in the literature on calculus of variations. The difference to the result stated in [9] is that the function $\phi$ is also dependent on the space variable $x$. Thus for the sake of completeness of the paper we include a proof although it is a straight forward modification of Theorem 1.1 in $[9]$.

Lemma 6. Let $\phi$ be uniformly Lipschitz continuous in $\Omega$ with respect to $\vec{w}$, i.e.,

$$
\left|\phi(x, \vec{w})-\phi\left(x, \vec{w}_{0}\right)\right| \leq L\left|\vec{w}-\vec{w}_{0}\right|
$$

Then the functional

$$
H(\vec{w}):=\int_{\Omega} \phi(x, \vec{w}) d x
$$

is weakly lower semi-continuous on $L^{p}\left(\Omega, \mathbb{R}^{d}\right)$ for any $1 \leq p<\infty$ if $\phi(x, \cdot)$ is convex for all $x \in \Omega$.

Proof. Let $\vec{w}_{n}$ be weakly convergent to $\vec{w}$ and $L:=\liminf _{n \rightarrow \infty} H\left(\vec{w}_{n}\right)$. We want to show that $L \geq H(\vec{w})$. Without loss of generality we assume that the sequence $\left\{\vec{w}_{n}\right\}$ satisfies

$$
L=\lim _{n \rightarrow \infty} H\left(\vec{w}_{n}\right) .
$$

From Mazur's lemma we get (see e.g. [11]) that there exists a sequence of convex combinations $\left\{\vec{v}_{n}\right\}$ such that

$$
\vec{v}_{n}=\sum_{k=n}^{N} \alpha_{k} \vec{w}_{k}, \text { where } \sum_{k=n}^{N} \alpha_{k}=1 \text { and } \alpha_{k} \geq 0 \text { and } n \leq k \leq N
$$


which converges to $\vec{w}$ in $L^{p}\left(\Omega, \mathbb{R}^{d}\right)$.

Using the Hölder-inequality and the Lipschitz continuity of $\phi$ we find

$$
\begin{aligned}
\left|H(\vec{w})-H\left(\vec{v}_{n}\right)\right| & \leq \int_{\Omega}\left|\phi(x, \vec{w})-\phi\left(x, \vec{v}_{n}\right)\right| d x \\
& \leq L \int_{\Omega}\left|\vec{w}-\vec{v}_{n}\right| d x \\
& \leq L(\operatorname{meas}(\Omega))^{(p-1) / p}\left(\int_{\Omega}\left|\vec{w}-\vec{v}_{n}\right|^{p} d x\right)^{1 / p}
\end{aligned}
$$

where we set $(\operatorname{meas}(\Omega))^{(p-1) / p}=1$ if $p=1$.

Thus for every $\varepsilon>0$, a sufficiently large $n$ exists such that

$$
H(\vec{w}) \leq H\left(\vec{v}_{n}\right)+\varepsilon
$$

Using the fact that $\phi$ is convex with respect to the second component we find for sufficiently large $N$

$$
\begin{aligned}
\int_{\Omega} \phi(x, \vec{w}) d x & \leq \int_{\Omega} \phi\left(x, \sum_{k=n}^{N} \alpha_{k} \vec{w}_{k}\right) d x+\varepsilon \\
& \leq \sum_{k=n}^{N} \alpha_{k} \int_{\Omega} \phi\left(x, \vec{w}_{k}\right) d x+\varepsilon \\
& \leq L+2 \varepsilon .
\end{aligned}
$$

Since $\varepsilon$ is arbitrary the assertion follows.

The assumption on the Lipschitz continuity in Lemma 6 can be modified: let us denote by $L_{x, \vec{w}, \vec{w}_{0}}$ the Lipschitz constant of $\phi$ at $x, \vec{w}$, and $\vec{w}_{0}$, i.e.,

$$
\left|\phi(x, \vec{w})-\phi\left(x, \vec{w}_{0}\right)\right| \leq L_{x, \vec{w}, \vec{w}_{0}}\left|\vec{w}-\vec{w}_{0}\right|
$$

If

$$
L_{x, \vec{w}, \vec{w}_{0}} \leq C\left(1+\max \left\{|\vec{w}|^{s},\left|\vec{w}_{0}\right|^{s}\right\}\right),
$$

with $0 \leq s \leq p-1$, holds, then the conclusion of (18), i.e., (19), remains valid.

Using Lemma 6 we are able to prove a result on lower semi-continuity on $B V(\Omega)$.

Theorem 7. Let $\phi(x, \vec{w})$ be convex with respect to $\vec{w}$. Moreover, let $\phi$ satisfy (21). Then for any uniformly bounded sequence $\left\{\vec{w}_{n}\right\}$ in $B V\left(\Omega, \mathbb{R}^{d}\right)$ with weak limit $\vec{w}$ in $L^{1}(\Omega)$ we have $\vec{w} \in B V\left(\Omega, \mathbb{R}^{d}\right)$ and

$$
I[\vec{w}] \leq \liminf _{n \rightarrow \infty} I\left[\vec{w}_{n}\right]
$$


Proof. The bounded variation semi-norm is weakly lower semi-continuous on $L^{1}\left(\Omega, \mathbb{R}^{d}\right)$, and thus $\vec{w} \in B V\left(\Omega, \mathbb{R}^{d}\right)$ satisfies

$$
\int_{\Omega}|\nabla \vec{w}|_{1} d x \leq \liminf _{n \rightarrow \infty} \int_{\Omega}\left|\nabla \vec{w}_{n}\right|_{1} d x<\infty
$$

This result follows from the fact that the dual of $L^{1}$ and $L^{\infty}$ are isomorphic, and therefore for each $\vec{v} \in C_{0}^{1}\left(\Omega, \mathbb{R}^{n}\right)$ and $i=1, \cdots, d$ we have

$$
\int_{\Omega} w^{i} \nabla \cdot \vec{v} d x=\lim _{n \rightarrow \infty} \int_{\Omega} w_{n}^{i} \nabla \cdot \vec{v} d x
$$

Consequently,

$$
\int_{\Omega}\left|\nabla w^{i}\right| d x=\sup _{\left\{\vec{v} \in C_{0}^{\infty}\left(\Omega, \mathbb{R}^{n}\right),|\vec{v}|_{L} \infty\left(\Omega, \mathbb{R}^{n}\right) \leq 1\right\}} \int_{\Omega} w^{i} \nabla \cdot \vec{v} d x \leq \liminf _{n \rightarrow \infty} \int_{\Omega}\left|\nabla w_{n}^{i}\right| d x,
$$

which shows the assertion.

The functional $H(\vec{w})$ is weakly lower semi-continuous on $L^{p}\left(\Omega, \mathbb{R}^{d}\right)$ for any $p \geq 1$ by Lemma 6 . Thus $I$ is weakly lower semi-continuous.

Under some additional assumptions we are even able to prove a lower semi-continuity result for nonconvex functionals which satisfy a growth condition.

Theorem 8. For $n=2,3$ let $\phi(x, \vec{w})$ be continuous and satisfy

$$
\left|\phi\left(x, \vec{w}_{n}\right)-\phi(x, \vec{w})\right| \leq L_{x, \vec{w}, \vec{w}_{n}}\left|\vec{w}_{n}-\vec{w}\right|
$$

with

$$
L_{x, \vec{w}, \vec{w}_{n}} \leq C\left(1+|\vec{w}|^{q}+\left|\vec{w}_{n}\right|^{q}\right)
$$

where $0 \leq q<1^{1}$ for $n=2$ and $0 \leq q<1 / 2$ for $n=3$. Then for any uniformly bounded sequence $\left\{\vec{w}_{n}\right\}$ in $B V\left(\Omega, \mathbb{R}^{d}\right)$ with weak limit $\vec{w}$ in $L^{1}(\Omega)$ we have

$$
I[w] \leq \liminf _{n \rightarrow \infty} I\left[w_{n}\right] .
$$

Proof. By means of the weak lower semi-continuity of the $B V$-seminorm we conclude that $\vec{w} \in B V\left(\Omega, \mathbb{R}^{d}\right)$ (see e.g. [17]).

- For $n=2$ each subsequence of $\left\{\vec{w}_{n}\right\}$ has a strongly convergent subsequence to $\vec{w}$ in $L^{s}\left(\Omega, \mathbb{R}^{d}\right)$ with $1 \leq s<2$. This follows from the compact Sobolev embedding theorem [20, Theorem 3.5.2 and Section 6.1.2]. Thus $\left\{\vec{w}_{n}\right\}$ is itself strongly convergent in $L^{s}\left(\Omega, \mathbb{R}^{d}\right)$. Moreover, the embedding of $B V\left(\Omega, \mathbb{R}^{d}\right)$ into $L^{2}\left(\Omega, \mathbb{R}^{d}\right)$

\footnotetext{
${ }^{1}$ All along this paper we use the convention $x^{0}=1$ for $x \geq 0$
} 
is bounded. Then by the Cauchy-Hölder inequality we get with the setting $t>2$ and $q t=2$ and $1 / s=1-1 / t$ (for $q=0$ set formally $t=\infty$ and use $x^{0}=1$ )

$$
\begin{aligned}
& \left|\int_{\Omega} \phi\left(x, \vec{w}_{n}\right)-\phi(x, \vec{w}) d x\right| \\
& \leq C \int_{\Omega} \max \left\{1+|\vec{w}|^{q}, 1+\left|\vec{w}_{n}\right|^{q}\right\}\left|\vec{w}-\vec{w}_{n}\right| d x \\
& \leq C\left(\max \left\{\int_{\Omega}\left(1+\left|\vec{w}_{n}\right|^{q}\right)^{t} d x, \int_{\Omega}\left(1+|\vec{w}|^{q}\right)^{t} d x\right\}\right)^{1 / t} \\
& \quad\left(\int_{\Omega}\left|\vec{w}-\vec{w}_{n}\right|^{s} d x\right)^{1 / s} .
\end{aligned}
$$

The last term tends to zero by the Sobolev embedding theorem.

- For $n=3$ each subsequence of $\left\{\vec{w}_{n}\right\}$ has a strongly convergent subsequence to $\vec{w}$ in $L^{s}\left(\Omega, \mathbb{R}^{d}\right)$ with $1 \leq s<3 / 2$. Thus it follows from the Cauchy-Hölder inequality with the setting $t>3 / 2$ and $q t=3 / 2$ and $1 / s=1-1 / t$ and the growth property of $\phi$ that

$$
\begin{aligned}
& \left|\int_{\Omega}\left(\phi\left(x, \vec{w}_{n}\right)-\phi(x, \vec{w})\right) d x\right| \\
& \leq C \int_{\Omega} \max \left\{\left(1+|\vec{w}|^{q}\right),\left(1+\left|\vec{w}_{n}\right|^{q}\right)\right\}\left|\vec{w}-\vec{w}_{n}\right| d x \\
& \leq C\left(\int_{\Omega} \max \left\{\left(1+|\vec{w}|^{q}\right)^{t},\left(1+\left|\vec{w}_{n}\right|^{q}\right)^{t}\right\} d x\right)^{1 / t} \\
& \quad\left(\int_{\Omega}\left|\vec{w}-\vec{w}_{n}\right|^{s} d x\right)^{1 / s} .
\end{aligned}
$$

Again from the Sobolev embedding theorem the assertion follows.

In particular the above proof reveals that the operator $H$ is even continuous on $B V\left(\Omega, \mathbb{R}^{d}\right)$.

\subsection{Quasiconvex functionals on $B D(\Omega)$}

Let $\Omega \subseteq \mathbb{R}^{n}$ be the space $B D(\Omega)$ of vector fields with bounded deformation (see e.g. [28]). This is the space of all vector fields $\vec{w} \in L^{1}\left(\Omega, \mathbb{R}^{n}\right)$ satisfying

$$
j(\vec{w})=\sum_{i, j=1}^{n} \int_{\Omega}\left|D_{i j} \vec{w}\right| d x<\infty ;
$$

here

$$
D_{i j}(\vec{w})=\frac{\partial w^{i}}{\partial x_{j}}+\frac{\partial w^{j}}{\partial x_{i}}
$$


has to be understood as a Radon measure.

We remark that analogously to BV-functions it can be shown that $j(\cdot)$ is weakly lower semi-continuous on $L^{p}\left(\Omega, \mathbb{R}^{d}\right), p \geq 1$.

Lemma 9. 1. Let $\left\{\vec{w}_{n}\right\}$ a sequence of functions in $B D(\Omega)$ which converges weakly in $\left(L^{p}(\Omega)\right)^{n}, 1 \leq p<\infty$ to $\vec{w}$, then

$$
j(\vec{w}) \leq j\left(\vec{w}_{n}\right)
$$

Due to similar difficulties as for the functions space $B V\left(\Omega, \mathbb{R}^{d}\right)$ we restrict our attention to functionals of the form

$$
J[w]:=\int_{\Omega} f\left(x, \vec{w}, D_{i j} \vec{w}\right) d x
$$

with

$$
f\left(x, \vec{w}, D_{i j}(\vec{w})\right)=\phi(x, \vec{w})+\sum_{i, j=1}^{d}\left|D_{i j}(\vec{w})\right| .
$$

Theorem 10. Let $\phi(x, \vec{w})$ satisfy the assumptions of Theorem 7. Then for any uniformly bounded sequence $\left\{\vec{w}_{m}\right\}$ in $B D(\Omega)$ with weak limit $\vec{w}$ in $L^{1}(\Omega)$ we have $\vec{w} \in$ $B D(\Omega)$ and

$$
I[\vec{w}] \leq \liminf _{m \rightarrow \infty} I\left[\vec{w}_{m}\right] .
$$

Proof. Temam and Strang [28] showed that the embedding

$$
i: B D(\Omega) \rightarrow\left(L^{n /(n-1)}(\Omega)\right)^{n}
$$

is continuous. Thus any bounded subsequence $\left\{\vec{w}_{m}\right\}$ in $B D(\Omega)$ has a weakly convergent subsequence in $\left(L^{n /(n-1)}(\Omega)\right)^{n}$ and the weak limit $\vec{w}$ is in $B D(\Omega)$ due to the weak lower semi-continuity of the $B D$-norm. This proves the assertion.

\section{Existence of minimizers of quasiconvex function- als}

The functional $G[\vec{w}]$ as introduced in (11) attains a minimum on any closed ball of $W^{1, p}\left(\Omega, \mathbb{R}^{d}\right), 1<p<\infty$ :

Theorem 11. Let $f$ satisfy (10) and be quasiconvex. Then $G[\vec{w}]$ attains a minimum on any closed ball of $W^{1, p}\left(\Omega, \mathbb{R}^{d}\right), 1<p<\infty$.

Proof. Let $\left\{\vec{w}_{n}\right\}$ be a sequence in a closed ball $\Theta$ of $W^{1, p}\left(\Omega, \mathbb{R}^{d}\right)$. Suppose that $G\left[\vec{w}_{n}\right]$ converges to $z$, the global minimum of $G[\vec{w}]$ in $\Theta$. By the theorem of Alaoglu-BourbakiKakutani, since $W^{1, p}\left(\Omega, \mathbb{R}^{d}\right)$ is reflexive, each ball is weakly compact and we can select a subsequence that is weakly convergent to $\vec{w} \in \Theta$ such that $G\left[\vec{w}_{n}\right] \rightarrow z$. Since $G$ is weakly lower semi-continuous the assertion follows. 
For the functional $I[\vec{w}]$ defined in $(7)$ we have the following results on existence of minimizers:

Theorem 12. Let $f: \bar{\Omega} \times \mathbb{R}^{d} \times \mathbb{R}^{d} \rightarrow[0, \infty)$ be continuous and $f(x, \vec{w}, \cdot)$ quasiconvex for all $(x, \vec{w}) \in \bar{\Omega} \times \mathbb{R}^{d}$.

1. Let $f$ additionally satisfy the assumptions item 2- 4 in Theorem 4 and

$$
\underline{f}(x)+\underline{\alpha}\left(|\vec{w}|^{p}+|P|^{p}\right) \leq f(x, \vec{w}, P)
$$

with $\underline{\alpha}>0$ and $\underline{f} \in L^{1}(\Omega)$, then $I[\vec{w}]$ attains a minimum on $W^{1, p}\left(\Omega, \mathbb{R}^{d}\right), 1<p<$ $\infty$.

2. Let $f$ additionally satisfy assumptions item 2- 4 in Corollary 5 and

$$
\underline{f}(x)+\underline{\alpha}\left(|\vec{w}|^{p}+|P|^{p}\right) \leq f(x, \vec{w}, P),
$$

with

$$
0<\underline{\alpha}, \quad \underline{f} \in L^{1}(\Omega) .
$$

Then $I[\vec{w}]$ attains a minimum on $W^{1, p}\left(\Omega, \mathbb{R}^{d}\right)$.

3. Let $f$ satisfy (17) and

$$
\underline{f}(x)+\underline{\alpha}|\vec{w}| \leq \phi(x, \vec{w}),
$$

where $f \in L^{1}(\Omega)$. If $\phi$ satisfies either the assumptions of Theorem 7 or Theorem 8 , then the functional $I[\vec{w}]$ attains a minimum in $B V\left(\Omega, \mathbb{R}^{d}\right)$.

4. Let $f$ satisfy (24) and (28). If $\phi$ satisfies the assumptions of Theorem 7, then the functional $J[\vec{w}]$ (defined in (23)) attains a minimum in $B D(\Omega)$.

Proof. 1.-2. The proof of the first and second item is very similar to the proof of Theorem 2.9 in [10, p.180].

3.-4. The proofs are similar to the proof of the first item by taking into account the special structure of the functionals $I[\vec{w}]$ and $J[\vec{w}]$.

\section{Analysis of optical flow models}

The optical flow models considered in (2) reveal a special structure which is inherent in many variational problems in nonlinear elasticity (see e.g. [5]). The most commonly used model are of the form

$$
f(x, \vec{w}, \nabla \vec{w})=\mathcal{W}(x, \nabla \vec{w})+\phi(x, \vec{w})
$$


In nonlinear elasticity $\mathcal{W}(x, \nabla \vec{w})$ is the stored-energy function and $\phi$ is a body force potential. The obvious coherence between nonlinear elasticity and optical flow models allows us to give physical interpretations in terms of nonlinear elastic models.

In the following we apply the general results of Sections 3 and 4 to the models outlined in Section 2.

All along this Section we restrict our attention to $\Omega=[0,1]^{2}$ and assume that the image data $I$ is in $C^{2}\left([0,1]^{2}\right)$, and we denote

$$
C:=\max \left\{\left|I_{x_{1}}(x)\right|,\left|I_{x_{2}}(x)\right|,\left|I_{t}(x)\right|,\left|I_{x_{i} x_{j}}(x)\right|,\left|I_{x_{1}, t}\right|: 1 \leq i, j \leq 2, x \in \bar{\Omega}\right\} .
$$

Let $\rho$ be a continuous non-negative function from $\mathbb{R}$ into $[0, \infty)$, then

$$
\phi(x, \vec{w}):=\rho\left((\nabla I)^{t}(x) \vec{w}+I_{t}(x)\right)+\underline{\alpha}|\vec{w}|^{p}
$$

is continuous with respect to $(x, \vec{w})$. The term $\underline{\alpha}|\vec{w}|^{p}$, with $\underline{\alpha}>0$, is used to guarantee ellipticity in the space $W^{1, p}\left(\Omega, \mathbb{R}^{d}\right)$ with $p>1$ or in $B V\left(\Omega, \mathbb{R}^{d}\right)$ with $1 \leq p \leq 2$. In most numerical simulations this term can be neglected since already the properties of $I$ guarantee that

$$
\rho\left((\nabla I)^{t}(x) \vec{w}+I_{t}(x)\right) \geq \underline{\alpha}|\vec{w}|^{p}
$$

However, in general it is not possible to derive this estimate as one sees from the trivial example $I=0$. In order to guarantee (30) one would have to impose technical assumptions on $I$ which we want to avoid.

In the following lemma we summarize a basic result on Lipschitz continuity of $\phi$.

Lemma 13. Let $\rho$ be uniformly Lipschitz continuous on every closed ball $\overline{B(r)} \subseteq \mathbb{R}$ with radius $r$, with Lipschitz constant $L_{r}$, i.e., for all $s_{1}, s_{2} \in \overline{B(r)}$ we have

$$
\left|\rho\left(s_{1}\right)-\rho\left(s_{2}\right)\right| \leq L_{r}\left|s_{1}-s_{2}\right|
$$

Let $\hat{r}=C(1+\sqrt{2}|\vec{w}|)$, then

$$
\left|\phi(x, \vec{w})-\phi\left(x_{0}, \vec{w}\right)\right| \leq L_{\hat{r}} C(\sqrt{2}+2|\vec{w}|)\left|x-x_{0}\right| .
$$

Moreover, with $\hat{r}=C\left(1+\sqrt{2} \max \left\{|\vec{w}|,\left|\vec{w}_{0}\right|\right\}\right)$ we have

$$
\left|\phi\left(x_{0}, \vec{w}\right)-\phi\left(x_{0}, \vec{w}_{0}\right)\right| \leq\left(\sqrt{2} L_{\hat{r}} C+\underline{\alpha p} \max \left\{|\vec{w}|^{p-1},\left|\vec{w}_{0}\right|^{p-1}\right\}\right)\left|\vec{w}-\vec{w}_{0}\right| .
$$

The following lemma relates convexity of $\rho$ and $\phi$ :

Lemma 14. Let $p \geq 1$ and $\rho$ convex. Then $\phi(x, \vec{w})$ is convex with respect to $\vec{w}$. 
Proof. For fixed $x \in \Omega$ the function

$$
h(\vec{w})=(\nabla I)^{t}(x) \vec{w}+I_{t}(x)
$$

is affine linear and thus the superposition

$$
\phi(x, \vec{w})=\rho \circ h(\vec{w})
$$

is convex (with respect to $\vec{w}$ ), too.

Now we are able to formulate an existence result in $B V\left(\Omega, \mathbb{R}^{2}\right)$ :

Theorem 15. - Let $1 \leq p<2$ in (29). If $\rho$ satisfies the growth condition

$$
L_{\hat{r}} \leq C_{L}\left(1+\hat{r}^{s}\right)
$$

with $0 \leq s<1$, then

$$
I[\vec{w}]=\int \phi(x, \vec{w}) d x+\alpha \int_{\Omega}|\nabla \vec{w}|_{1} d x
$$

attains a minimum on $B V\left(\Omega, \mathbb{R}^{2}\right)$.

- For $1 \leq p \leq 2$ in (29). If $\rho$ is convex with respect to $\vec{w}$, and satisfies (33) with $0 \leq s \leq 1$, then $I[\vec{w}]$ attains a minimum on $B V\left(\Omega, \mathbb{R}^{2}\right)$.

Proof. Let $q:=\max \{s, p-1\}$, then from (32) we get

$$
\left|\phi(x, \vec{w})-\phi\left(x, \vec{w}_{0}\right)\right| \leq \tilde{C}\left(1+\max \left\{|w|^{q},\left|w_{0}\right|^{q}\right\}\right)\left|\vec{w}-\vec{w}_{0}\right| .
$$

1. In the first case the function $\phi$ satisfies the general assumption of Theorem 8. For $p=1,(28)$ is trivially satisfied with $0=\underline{c}=\underline{f}$. For $p>1$,

$$
\underline{\alpha}|\vec{w}|^{p}=\underline{\alpha}\left(1+|\vec{w}|^{p}\right)-\underline{\alpha} \geq \underline{\alpha}|\vec{w}|-\underline{\alpha} .
$$

Thus, (28) holds with $\underline{f}=\underline{c}=-\underline{\alpha}$. From Theorem 12 the assertion follows.

2. The embedding of $B V\left(\Omega, \mathbb{R}^{d}\right)$ in $L^{2}\left(\Omega, \mathbb{R}^{d}\right)$ is bounded. Let $I\left[w_{n}\right]$ converge to its infimum, then due to the boundedness of $\left\{w_{n}\right\}$ in $B V\left(\Omega, \mathbb{R}^{d}\right)$ it has a subsequence which is weakly convergent in $L^{2}\left(\Omega, \mathbb{R}^{d}\right)$. From Lemma 6 it follows (taking into account the remark after this lemma) that $\int_{\Omega} \phi(x, \vec{w}) d x$ is weakly lower semicontinuous on $L^{2}\left(\Omega, \mathbb{R}^{d}\right)$. Since the bounded variation seminorm is weakly lower semi-continuous on $B V\left(\Omega, \mathbb{R}^{d}\right)$ as well, the assertion follows. 
Now we turn to existence results on $W^{1, p}\left(\Omega, \mathbb{R}^{d}\right)$. For $1<p<2$, let $1 \leq q \leq \frac{2 p}{2-p}$, for $p \geq 2$ let $1 \leq q<\infty$. Set $\bar{s}=\max \{p, q\}$ and let $\rho$ be nonnegative with $\rho(0)=0$, satisfying

$$
L_{\hat{r}} \leq C_{L}\left(1+\hat{r}^{\bar{s}-1}\right)
$$

Then we have

$$
\begin{aligned}
0 & \leq \phi(x, \vec{w}) \\
& =\rho\left((\nabla I)^{t}(x) \vec{w}+I_{t}(x)\right)-\rho(0)+\underline{\alpha}|\vec{w}|^{p} \\
& \leq \tilde{C}\left(1+|\vec{w}|^{\bar{s}}\right),
\end{aligned}
$$

with a generic constant $\tilde{C}$. This in particular shows that item 2 in Corollary 5 holds with $f$ replaced by $\phi+\mathcal{W}$ if $\mathcal{W}(x, \nabla \vec{w})$ quasiconvex, satisfying

1. there exists a positive constant $\beta>0$ such that

$$
\left|\mathcal{W}\left(x, P_{1}\right)-\mathcal{W}\left(x, P_{2}\right)\right| \leq \beta\left(1+\left|P_{1}\right|^{p-1}+\left|P_{2}\right|^{p-1}\right)\left|P_{1}-P_{2}\right|,
$$

2. there exists a continuous, increasing function $\eta$ satisfying $\eta(0)=0$ such that

$$
|\mathcal{W}(x, P)-\mathcal{W}(y, P)| \leq \eta(|x-y|)\left(1+|P|^{p}\right),
$$

3. there exist constants $\underline{\alpha}>0, \bar{\alpha}>0$, and $\underline{c} \in \mathbb{R}$, such that

$$
\underline{c}+\underline{\alpha}\left(1+|P|^{p}\right) \leq \mathcal{W}(x, P) \leq \bar{\alpha}\left(1+|P|^{p}\right) .
$$

From (31) and (32) we see that items 3 and 4 in Corollary 5 hold with $f$ replaced by $\phi+\mathcal{W}$. Moreover, (27) holds by the imposed assumptions on $\mathcal{W}$ and (29). Thus according to Theorem 12 the functional $I[\vec{w}]$ with $\rho$ satisfying (34) attains a minimum in $W^{1, p}\left(\Omega, \mathbb{R}^{2}\right)$.

\subsection{Examples}

In the following we show particular examples of weakly lower semi-continuous optical flow models.

1. Let $1 \leq s+1 \leq 2$. The function $\rho(\cdot)=|\cdot|^{s+1}$ is convex and Lipschitz continuous on bounded sets and satisfies

$$
L_{\hat{r}} \leq p \hat{r}^{s} .
$$

Thus according to Lemma 14 and Theorem 15 the functional

$$
I[\vec{w}]:=\int_{\Omega}\left|(\nabla I)^{t} \vec{w}+I_{t}\right|^{s+1} d x+\alpha \int_{\Omega}\left(\underline{\alpha}|\vec{w}|+|\nabla \vec{w}|_{1}\right) d x,
$$

attains a minimum on $B V\left(\Omega, \mathbb{R}^{d}\right)$. 
The case $s=0$ has been studied in [3].

2. Let us consider the specific example

$$
\int_{\Omega}\left|(\nabla I)^{t}(x) \vec{w}+I_{t}(x)\right|^{q} d x+\alpha\|\vec{w}\|_{W^{1, p}\left(\Omega, \mathbb{R}^{d}\right)}^{p},
$$

with $1<p$ and $q$ satisfying (16) (with $n=2$ ).

The according function $\rho(t)=t^{q}$ is Lipschitz continuous with Lipschitz constant $L_{\hat{r}}=C\left(1+\hat{r}^{q-1}\right)$. Thus from Theorem 12 and Corollary 5 it follows that $I[\vec{w}]$ attains a minimum on $W^{1, p}\left(\Omega, \mathbb{R}^{2}\right)$.

The case $q=1, p=2$ has been studied in [18].

The case $q=2, p=2$ goes back to [19] and has been analyzed in [26].

As long as $\mathcal{W}(P)$ is quasiconvex, satisfies some growth rate and is elliptic, Theorem 12 and Corollary 5 are valid and guarantee weak lower semi-continuity of $I[\vec{w}]$ on $W^{1, p}\left(\Omega, \mathbb{R}^{d}\right)$ and existence of a minimizer.

In particular the general results are applicable in the following situations:

Let $p=2,1 \leq q$ and $\rho$ satisfy

$$
L_{\hat{r}} \leq \hat{c}\left(1+\hat{r}^{q-1}\right)
$$

- With

$$
\mathcal{W}(P)=\left|P_{1}\right|^{2}+\left|P_{2}\right|^{2}
$$

the functional $I[\vec{w}]$ is weakly lower semi-continuous on $W^{1,2}\left(\Omega, \mathbb{R}^{2}\right)$ and attains a minimum.

- For the integrand in the anisotropic diffusion penalizing functional (4) there exist constants $c, C$ such that

$$
c\left(\left|\nabla P_{1}\right|^{2}+\left|\nabla P_{2}\right|^{2}\right) \leq \mathcal{W}(x, P) \leq C\left(\left|\nabla P_{1}\right|^{2}+\left|\nabla P_{2}\right|^{2}\right)
$$

for all $P \in \mathbb{R}^{2 \times 2}$. Investigating the eigenvalues of $D_{2}(\nabla I)$ shows that one may choose

$$
\begin{aligned}
c & :=\frac{\lambda^{2}}{\sup _{x}|\nabla I(x)|^{2}+2 \lambda^{2}} \\
C & :=1
\end{aligned}
$$

This shows (37). The functional $\mathcal{W}(x, \cdot)$ is quasiconvex since it is a sum of convex operations. The estimates (35) and (36) follow from elementary calculus. 
- Let $\tau=\left|P_{1}\right|^{2}+\left|P_{2}\right|^{2}$, then the function

$$
\phi_{i}(\tau)=\epsilon \tau+\lambda_{i}^{2} \sqrt{1+\tau / \lambda_{i}^{2}}-\lambda_{i}^{2}
$$

is convex with respect to $\left(P_{1}, P_{2}\right)$ and satisfies (35)-(37); here $\epsilon>0$ is a positive parameter and $\lambda_{i}>0$ denotes a contrast parameter.

3. Nonconvex growth functions may be considered as well:

$$
\int_{\Omega} \log \left(1+\left|(\nabla I)^{t}(x) \vec{w}+I_{t}(x)\right|^{2}\right) d x+\alpha\|\vec{w}\|_{W^{1, p}\left(\Omega, \mathbb{R}^{d}\right)}^{p},
$$

with $1<p<\infty$ attains a minimum on $W^{1, p}\left(\Omega, \mathbb{R}^{d}\right)$.

$$
\int_{\Omega} \log \left(1+\left|(\nabla I)^{t}(x) \vec{w}+I_{t}(x)\right|^{2}\right) d x+\alpha\|\vec{w}\|_{B V\left(\Omega, \mathbb{R}^{d}\right)},
$$

attains a minimum on $B V\left(\Omega, \mathbb{R}^{d}\right)$.

4. $|\cdot|$ is convex and thus from Lemma 14 we see that $\phi(x, \cdot)$ is convex, too. Thus from Theorem 12 it follows that

$$
\int_{\Omega}\left|(\nabla I)^{t}(x) \vec{w}+I_{t}(x)\right| d x+\alpha j(\vec{w})+\underline{\alpha} \int_{\Omega}|\vec{w}|_{L^{1}(\Omega)} d x
$$

attains a minimum on $B D(\Omega)$.

\section{Numerical Experiments}

For our numerical experiments we consider the non-quadratic functional

$$
E(\vec{w})=\int_{\Omega}\left(\phi_{1}\left(\left|(\nabla I)^{t}(x) \vec{w}+I_{t}(x)\right|^{2}\right)+\alpha \phi_{2}\left(|\nabla \vec{w}|^{2}\right) d x\right.
$$

where the growth function $\phi_{i}$ was specified in (39). In order to show the influence of the growth function on the data term we compare this functional with [30]

$$
E(\vec{w})=\int_{\Omega}\left(\left|(\nabla I)^{t}(x) \vec{w}+I_{t}(x)\right|^{2}+\alpha \phi_{2}\left(|\nabla \vec{w}|^{2}\right) d x\right.
$$

For both convex functionals, the steepest descent equations have been discretized with a simple explicit finite difference scheme.

The results are depicted in Figure 1. It shows a well-known test sequence with a taxi scene. Using functional (41) leads to a relatively noisy optical flow field. With functional (40), noise is successfully removed and the flow field of the taxi is more homogeneous and realistic. For a suitable parameter choice, it is even possible to focus on the taxi movement by smoothing away the flow fields of the two faster vehicles. Since they were 
faster, they are treated as outliers in the data term. As a result, they are significantly less penalized than in the functional (41).

This shows that from a practical viewpoint, it may be interesting to consider nonquadratic growth functions not only in the regularizer, but also in the data term. They may lead to increased robustness and give additional degrees of freedom. Experiments by Black and Ananden $[6,7]$ point in the same direction. In their articles, nonconvex growth functionals have been motivated from robust statistics. However, our numerical experiments indicate that a similar effect might be achieved using convex non-quadratic growth functionals (40) which are more convenient from a numerical point of view.
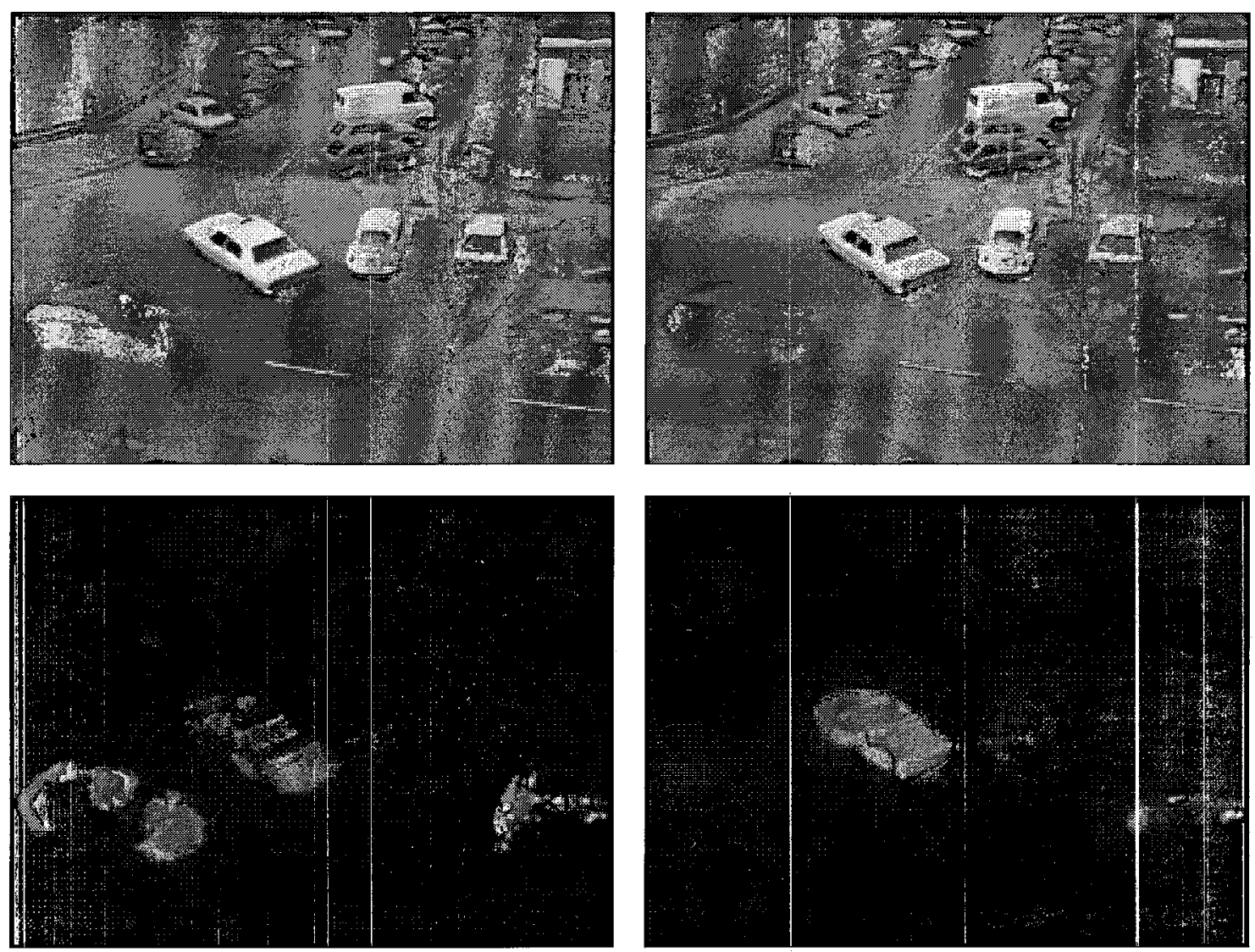

Figure 1: (a) Top LefT: Frame 10 of the Hamburg taxi sequence, $\Omega=(0,256) \times$ $(0,190)$. (b) Top Right: Frame 11. The car in the lower left corner moved to the right, the taxi turned around the street corner, and the bus at the lower right corner moved to the left. (c) BotTom LeFT: Computed optic flow magnitude using the functional (41) with the parameters $\alpha=1000$ and $\lambda_{2}=0.01$. (d) BotTom Right: Result using (40) with $\alpha=1000, \lambda_{1}=0.000001$, and $\lambda_{2}=0.01$. 


\section{References}

[1] R.A. Adams. Sobolev Spaces. Academic Press, New York, 1975.

[2] L. Alvarez, J. Weickert, and J. Sanchez. A scale-space approach to nonlocal optical flow calculations. In [24], pages 235-246, 1999.

[3] G. Aubert, R. Deriche and P. Kornprobst, Computing optical flow via variational techniques. SIAM J. Appl. Math., 60:156-182, 1999.

[4] P. Aviles and Y. Giga. Variational integrals on mappings of bounded variation and their lower semicontinuity. Arch. Ration. Mech. Anal., 115(3):201-255, 1991.

[5] J.M. Ball. Convexity conditions and existence theorems in nonlinear elasticity. Arch. Rat. Mech. Anal., 63:337--403, 1977.

[6] M. J. Black and P. Anandan. Robust dynamic motion estimation over time. In Proc. IEEE Comp. Soc. Conf. on Computer Vision and Pattern Recognition (CVPR '91, Maui, June 3-6, 1991), IEEE Computer Society Press, Los Alamitos, pages 292302, 1991 .

[7] M. J. Black and P. Anandan. The robust estimation of multiple motions: Parametric and piecewise-smooth flow fields. Computer Vision and Image Understanding, 63:75-104, 1996.

[8] I. Cohen, Nonlinear variational method for optical flow computation. In Proc. Eighth Scandinavian Conf. on Image Analysis (SCIA '93, Tromsø, May 25-28, 1993), Vol. 1, pages 523-530, 1993.

[9] B. Dacorogna. Weak Continuity and Weak Lower Semi-continuity of Non-linear Functionals. Springer-Verlag, Berlin, 1982.

[10] B. Dacorogna. Direct Methods in the Calculus of Variations. Springer-Verlag, Berlin, 1989.

[11] I. Ekeland and R. Temam. Convex Analysis and Variational Problems. North Holland, Amsterdam, 1976.

[12] L. C. Evans. Weak Convergence Methods for Nonlinear Partial Differential Equations. American Mathematical Society, Providence, RI, 1990.

[13] L.C. Evans and R.F. Gariepy. Measure Theory and Fine Properties of Functions. CRC-Press, Boca Raton, 1992.

[14] I. Fonseca and A. Francfort. Relaxation of BV versus quasiconvexification in $W^{1, p}$; a model for the interaction between fracture and damage. Calc. Var., 3:407-446, 1995.

[15] I. Fonseca and St. Müller. Quasi-convex integrands and lower semicontinuity in $L^{1}$. SIAM J. Math. Anal., 23:1081-1098, 1992. 
[16] I. Fonseca and St. Müller. Relaxation of quasiconvex functionals in $B V\left(\Omega, R^{p}\right)$ for integrands $f(x, u, \nabla u)$. Arch. Ration. Mech. Anal., 123:1-49, 1993.

[17] E. Giusti. Minimal Surfaces and Functions of Bounded Variation. Birkhäuser, Boston, 1984.

[18] W. Hinterberger. Generierung eines Films zwischen zwei Bildern mit Hilfe des optischen Flusses, 2000. Master's thesis, Johannes-Kepler Universität Linz, Austria.

[19] B. Horn and B. Schunck. Determining optical flow. Artif. Intell., 17:185-203, 1981.

[20] V. G. Maz'ya. Sobolev Spaces. Springer-Verlag, Berlin, 1985.

[21] Ch. Morrey. Quasi-convexity and the lower semicontinuity of multiple integrals. Pac. J. Math., 2:25-53, 1952.

[22] Ch. Morrey. Multiple Integrals in the Calculus of Variations. Springer-Verlag, New York, 1966.

[23] H.-H. Nagel. On the estimation of optical flow: relations between new approaches and some new results. Artif. Intell., 33:299-324, 1987.

[24] M. Nielsen, P. Johansen, O.F. Olsen, and J. Weickert, editors. Scale-Space Theories in Computer Vision. Lecture Notes in Computer Science Vol. 1683, Springer Verlag, 1999. Proceedings of the Second International Conference, Scale-Space'99, Corfu, Greece, 1999.

[25] S. Orphanoudakis, P. Trahanias, J. Crowley, and N. Katevas, editors. Proc. Computer Vision and Mobile Robotics Workshop. CVMR' 98, Santorini, Greece, Sept. 1998.

[26] C. Schnörr. Determining optical flow for irregular domains by minimizing quadratic functionals of a certain class. Int. J. of Comp. Vision, 6(1):25-38, 1991.

[27] C. Schnörr. Segmentation of visual motion by minimizing convex non-quadratic functionals. In Proc. 12th Int. Conf. on Pattern Recognition, Jerusalem, Israel, Vol. A, pages 661-663, Oct. 1994.

[28] R. Temam and G. Strang. Functions of bounded deformation. Arch. Ration. Mech. Anal., 75:7-21, 1980.

[29] J. Weickert. Anisotropic Diffusion in Image Processing. Teubner, Stuttgart, 1998.

[30] J. Weickert. On discontinuity-preserving optic flow. In [25], pages 115-252, 1998.

[31] J. Weickert and Ch. Schnörr. Variational optic flow computation with a spatiotemporal smoothness constraint. J. Math. Imag. Vision, in press. 